\title{
Geometric Analysis of Bifurcation and Symmetry Breaking in a Gross-Pitaevskii equation
}

\author{
R.K. Jackson ${ }^{* \dagger}$ and M.I. Weinstein ${ }^{\ddagger}$
}

October 29, 2018

Dedicated to Elliott H. Lieb on the occasion of his seventieth birthday

\begin{abstract}
Gross-Pitaevskii and nonlinear Hartree equations are equations of nonlinear Schrödinger type, which play an important role in the theory of Bose-Einstein condensation, Recent results of Aschenbacher et al [3] demonstrate, for a class of 3 - dimensional models, that for large boson number (squared $L^{2}$ norm), $\mathcal{N}$, the ground state does not have the symmetry properties as the ground state at small $\mathcal{N}$. We present a detailed global study of the symmetry breaking bifurcation for a 1 - dimensional model Gross-Pitaevskii equation, in which the external potential (boson trap) is an attractive double-well, consisting of two attractive Dirac delta functions concentrated at distinct points. Using dynamical systems methods, we present a geometric analysis of the symmetry breaking bifurcation of an asymmetric ground state and the exchange of dynamical stability from the symmetric branch to the asymmetric branch at the bifurcation point.
\end{abstract}

\section{Introduction}

The experimental realization of Bose-Einstein condensation has given great impetus to the study of the Gross-Pitaevskii and nonlinear Hartree equations. These are equations of nonlinear Schrödinger (NLS) type, with a potential having both linear and nonlinear parts. Such equations arise in the study of large dilute systems of bosons; $N$-body quantum mechanics of coupled bosons, where $N$ tends to infinity and the coupling strength tends to zero, 4, 11, 5] 1]. Of fundamental interest are the properties of the ground state of such systems.

\footnotetext{
*supported by a grant from the National Science Foundation under award number DMS0202542

${ }^{\dagger}$ Department of Mathematics and Statistics, Boston University

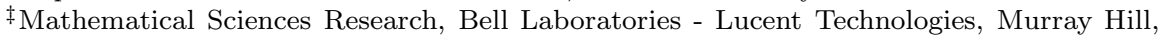
$\mathrm{NJ}$
} 
Because of the nonlinearity, the ground state can exhibit non-trivial transitions in structure.

Indeed, that such a transition takes place was established in the recent paper [3. They consider the nonlinear Hartree energy functional

$$
H_{g}[\Psi, \bar{\Psi}]=\frac{1}{2} \int_{\mathbb{R}^{d}}|\nabla \Psi(x)|^{2}+v(x)|\Psi(x)|^{2}+g|\Psi(x)|^{2}\left(V *|\Psi|^{2}\right)(x) \mathrm{d} x .
$$

Here, $v(x)$ is an external potential (the boson trap), and $V(x)$ is the two-body Coulomb interaction potential $V(x)=|x|^{-1}$ between bosons. The ground state of a system of $\mathcal{N}$ bosons is characterized by solution to the minimization problem

$$
\inf \left\{H_{g}[\Psi, \bar{\Psi}]:\|\Psi\|_{2}^{2}=\mathcal{N}\right\} .
$$

For spatial dimensions $d \geq 2$ and the case in which $g<0$ is fixed, corresponding to attractive interatomic forces (negative scattering length), it is shown that for a class of potentials $v(x)$, e.g., the double well potential, that if $\mathcal{N}$ is sufficiently large, then symmetry breaking in the ground state occurs, in the sense that the ground state of (1.2) does not have the same symmetries as $v(x)$. The proof in [3] is based upon the intuition in the double-well case, for example, that for small amplitude states $(\mathcal{N}$ small $)$ the nonlinear potential is negligible, and therefore the ground state should be a small distortion of the linear ground state $(g=0)$, and have the same symmetries as the linear ground state. However, by careful construction of trial functions, one can see that for $\mathcal{N}$ sufficiently large, $\mathcal{N}>\mathcal{N}_{\text {thresh }}$, it is energetically preferable for the ground state to be concentrated in one well or the other, but not equally in both.

The size of $\mathcal{N}_{\text {thresh }}$ and the dynamical stability properties of the various coexisting states (symmetric and asymmetric) is unaddressed. In this work we address these questions in the context of the simpler model, the one-dimensional Gross-Pitaevskii equation (GP/NLS)

$$
\mathrm{i} \psi_{t}=-\psi_{x x}-2|\psi|^{2} \psi+\epsilon v(x) \psi
$$

where $v(x)$ denotes the attractive double well potential

$$
v(x)=-\delta(x-L)-\delta(x+L),
$$

with conserved Hamiltonian energy functional

$$
\mathcal{H}_{\mathrm{GP}}[\psi, \bar{\psi}]=\int\left(\left|\frac{\mathrm{d} \psi(x)}{\mathrm{d} x}\right|^{2}+v(x)|\psi(x)|^{2}-\frac{1}{2}|\psi(x)|^{4}\right) \mathrm{d} x
$$

and conserved particle (boson) number

$$
\mathcal{N}[\psi, \bar{\psi}]=\int|\psi(x)|^{2} \mathrm{~d} x .
$$

Symmetry breaking bifurcations have been observed in this model [6] and in the analogous double square well model [12, by piecewise analytical solution in 
terms of hyperbolic secant and Jacobi elliptic function solutions of the translation invariant NLS equation $(v=0)$, followed by numerical solution of the nonlinear algebraic equations resulting from the required continuity and jump conditions at singular points of the equation's coefficients.

We present a simple geometric analysis demonstrating

(A) the symmetry breaking of the ground state, as a bifurcation of an asymmetric branch of solutions from the branch of symmetric solutions

and

(B) the exchange of dynamical stability from the symmetric branch to the asymmetric branch. In particular, beyond the bifurcation point the symmetric state is linearly exponentially and nonlinearly unstable, whereas the asymmetric state is nonlinearly orbitally stable.

We note that our analysis does not require the specific cubic nonlinearity and is applicable for general local nonlinearities, where $|\psi|^{2} \psi$ is replaced by $f\left(|\psi|^{2}\right) \psi$, with $f(\cdot)$ of a general class.

A bifurcation diagram showing the symmetric, antisymmetric and asymmetric states is presented in figure 1 The solid curve corresponds to the nonlinearly stable ground state. As $\lambda$ is increased $\left(-\lambda^{2}\right.$ is the frequency) the symmetric solution becomes unstable at the bifurcation point, while the new asymmetric state is nonlinearly stable. We expect that anti-symmetric states (nonlinear excited states), corresponding to points on the disconnected branch to the left of the symmetric branch, are unstable to generic perturbations due to a nonlinear resonance phenomena, and that for large $t$, the system's energy resides in the ground state and radiation modes; see, for example, 14, 15].

\section{Overview of Gross-Pitaevskii / NLS}

In appendix $\mathrm{A}$ we review the well-posedness theory of (1.31.4). The initial value problem is well-posed in $C^{0}\left(R^{1} ; H^{1}\left(R^{1}\right)\right)$.

In this section we focus on nonlinear bound states of (1.3). These are solutions of the form:

$$
\psi_{b}(x, t)=\mathrm{e}^{\mathrm{i} \lambda^{2} t} \psi(x ; \lambda), \psi \in L^{2} .
$$

For the linear Schrödinger equation, the functions $\psi$ are eigenstates of a Schrödinger operator: $-\partial_{x}^{2}+v(x)$ and satisfy the equation

$$
-\psi_{x x}+v(x) \psi=-\lambda^{2} \psi .
$$

In the case where $v(x)$ is given by (1.4), with $L$ sufficiently large, the set of bound states is two-dimensional, spanned by symmetric and antisymmetric functions. 


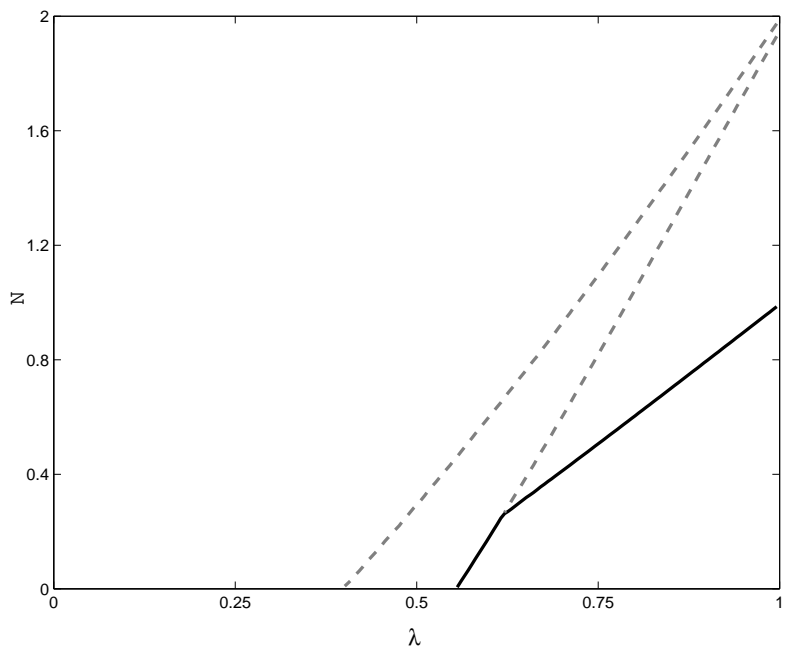

Figure 1: A bifurcation diagram is given for the case $\epsilon=1, L=2$. The curve on the left bifurcating from $\mathcal{N}=0$ represents the antisymmetric pulse, whereas the curve on the right represents the symmetric pulse. At the threshold value $\mathcal{N}_{\text {thresh }}$, an asymmetric pulse bifurcates from the symmetric pulse. At $\mathcal{N}-$ levels where the asymmetric pulse is present, the symmetric pulse is unstable.

For (1.3), nonlinear bound states satisfy the equation

$$
-\psi_{x x}+\left(v(x)-2|\psi|^{2}\right) \psi=-\lambda^{2} \psi .
$$

For the translation-invariant case, $v=0$, there is a well-known family of solitary ("soliton") traveling wave solutions. These are Galilean boosts (see (A.2) of the basic solitary ("soliton") standing wave with hyperbolic secant spatial profile. For a non-trivial potential $v(x)$ as in (1.4), the equation is no longer translation-invariant and we have "defect" or "pinned" states. These pinned nonlinear bound states are plotted in the bifurcation diagram of figure 1 In the translation invariant case, $v \equiv 0$, the nonlinear bound states reduce to the branch of NLS solitons, bifurcating from the zero state at zero frequency, the endpoint of the continuous spectrum of the linearized operator $-\partial_{x}^{2}$ about the zero state. For $v(x)$ nontrivial, there is a family of defect states, bifurcating from the zero state at each linear eigenfrequency of the linearized operator, $-\partial_{x}^{2}+v(x)$ and in the direction of the corresponding eigenfunction; see [13] for a general discussion.

Stability of nonlinear bound states: An important characterization of the nonlinear bound states is variational. The advantage of the variational characteri- 
zation is that it can be used to establish nonlinear stability of the ground state; see [18, 13.

Theorem 1. (I) Nonlinear bound states can be constructed variationally by minimizing the Hamiltonian, $\mathcal{H}$, subject to fixed $L^{2}$-norm, $\mathcal{N}$ :

$$
\min _{\varphi} \mathcal{H}[\varphi], \quad \mathcal{N}[\varphi]=\rho .
$$

Such minimizers are called nonlinear ground states, $\psi_{\text {Def }}(\rho)$. The associated frequency, $-\lambda^{2}(\rho)$, arises as a Lagrange multiplier for the constrained variational problem (2.4). As $\rho \rightarrow 0,-\lambda^{2}(\rho)$ tends to the ground state eigenvalue of $-\partial_{x}^{2}+v(x)$.

(II) Ground states are $H^{1}$ nonlinearly orbitally Lyapunov stable, i.e., if the initial data are $H^{1}$ close to a soliton (modulo the phase and translation symmetries of $G P / N L S)$, then the solution remains close to a soliton in this sense for all $t \in(-\infty, \infty)$.

Although, the above theorem establishes the ground state as a dynamically stable state, it leaves unaddressed the question of detailed spatial characteristics of the ground state. As we shall now see, dynamical systems methods can, in some cases, be used to study this question globally and in detail.

\section{Nonlinear bound states - dynamical systems approach}

Dynamical systems methods are particularly well-suited to studying the existence and nature of nonlinear bound states for the one-dimensional problem (1.31.4), since the equation is piecewise autonomous. In particular, we study

$$
-\psi_{x x}+\epsilon v(x) \psi-2 \psi^{3}=-\lambda^{2} \psi
$$

where $v(x)=-\delta(x+L)-\delta(x-L)$. With a quick apology for the following non-physical substitutions, we rescale the variables as follows:

$$
\begin{aligned}
x & =z / \lambda \\
\psi(x) & =\lambda \phi(\lambda x)=\lambda \phi(z) .
\end{aligned}
$$

In these variables, we have

$$
\phi_{z z}=\phi-2 \phi^{3}-\frac{\epsilon}{\lambda^{2}} v(z / \lambda) \phi .
$$

This is equivalent to the following system with matching conditions:

$$
\begin{array}{cc}
z \neq \pm \lambda L & \phi^{\prime \prime}=\phi-2 \phi^{3} \\
z= \pm \lambda L & \left\{\begin{array}{l}
\phi\left(z^{+}\right)=\phi\left(z^{-}\right) \\
\phi^{\prime}\left(z^{+}\right)=\phi^{\prime}\left(z^{-}\right)-\frac{\epsilon}{\lambda} \phi(z),
\end{array}\right.
\end{array}
$$


where ' represents differentiation with respect to $z$ and $f\left(z^{ \pm}\right)=\lim _{\rho \rightarrow 0} f(z \pm \rho)$. As usual, we can convert a second order differential equation into a pair of first order equations. In particular, if we let $(u, v)=\left(\phi, \phi^{\prime}\right)$, then we have

$$
\begin{array}{ll}
z \neq \pm \lambda L & \left\{\begin{array}{l}
u^{\prime}=v \\
v^{\prime}=u-2 u^{3}
\end{array}\right. \\
z= \pm \lambda L & \left\{\begin{array}{l}
u\left(z^{+}\right)=u\left(z^{-}\right) \\
v\left(z^{+}\right)=v\left(z^{-}\right)-\frac{\epsilon}{\lambda} u(z) .
\end{array}\right.
\end{array}
$$

A search for bound states (standing waves) in this system can then be undertaken as follows:

1. Since any standing wave must decay as $z \rightarrow-\infty$, a potential standing wave solution must originate (in the phase plane) along the global unstable manifold of $(0,0)$. Without loss of generality, we consider only the portion of this manifold that lies in the first quadrant.

2. When this solution approaches the first well at $z=-\lambda L^{-}$, we may shift $z$ so that the point $\lim _{z \rightarrow-\lambda L^{-}}(u(z), v(z))$ can be chosen anywhere along the unstable manifold. (The value of $u(-\lambda L)$ can be thought of as a shooting parameter.) At this chosen point, the solution jumps vertically according to the matching conditions for $v$.

3. After completing the vertical jump at $z=-\lambda L$, we again flow the solution forward for "time" $2 \lambda L$ according to the ordinary differential equation, until we approach the second well at $z=+\lambda L$.

4. When $z=+\lambda L$, the solution again jumps vertically in the phase plane according to the matching condition. The phase plane solution that we have been following represents a standing wave if and only if this second jump lands our solution exactly upon the stable manifold, in accordance with the condition that $u(z) \rightarrow 0$ as $z \rightarrow+\infty$.

For fixed values of the parameters $\epsilon, \lambda$ and $L$, it may happen that there are multiple standing waves present, corresponding to different values of the function at the first defect. In accordance with the search method described above, such solutions are shown in the phase plane in figure 2

\subsection{The linear limit; infinitesimal solutions}

To illuminate this search for standing waves, it is instructive to first consider the case of small amplitude solutions. For infinitesimal solutions, we neglect the nonlinear term and we are left with the following linear system

$$
\begin{array}{ll}
z \neq \pm \lambda L & \left\{\begin{array}{l}
u^{\prime}=v \\
v^{\prime}=u
\end{array}\right. \\
z= \pm \lambda L & \left\{\begin{array}{l}
u\left(z^{+}\right)=u\left(z^{-}\right) \\
v\left(z^{+}\right)=v\left(z^{-}\right)-\frac{\epsilon}{\lambda} u(z) .
\end{array}\right.
\end{array}
$$



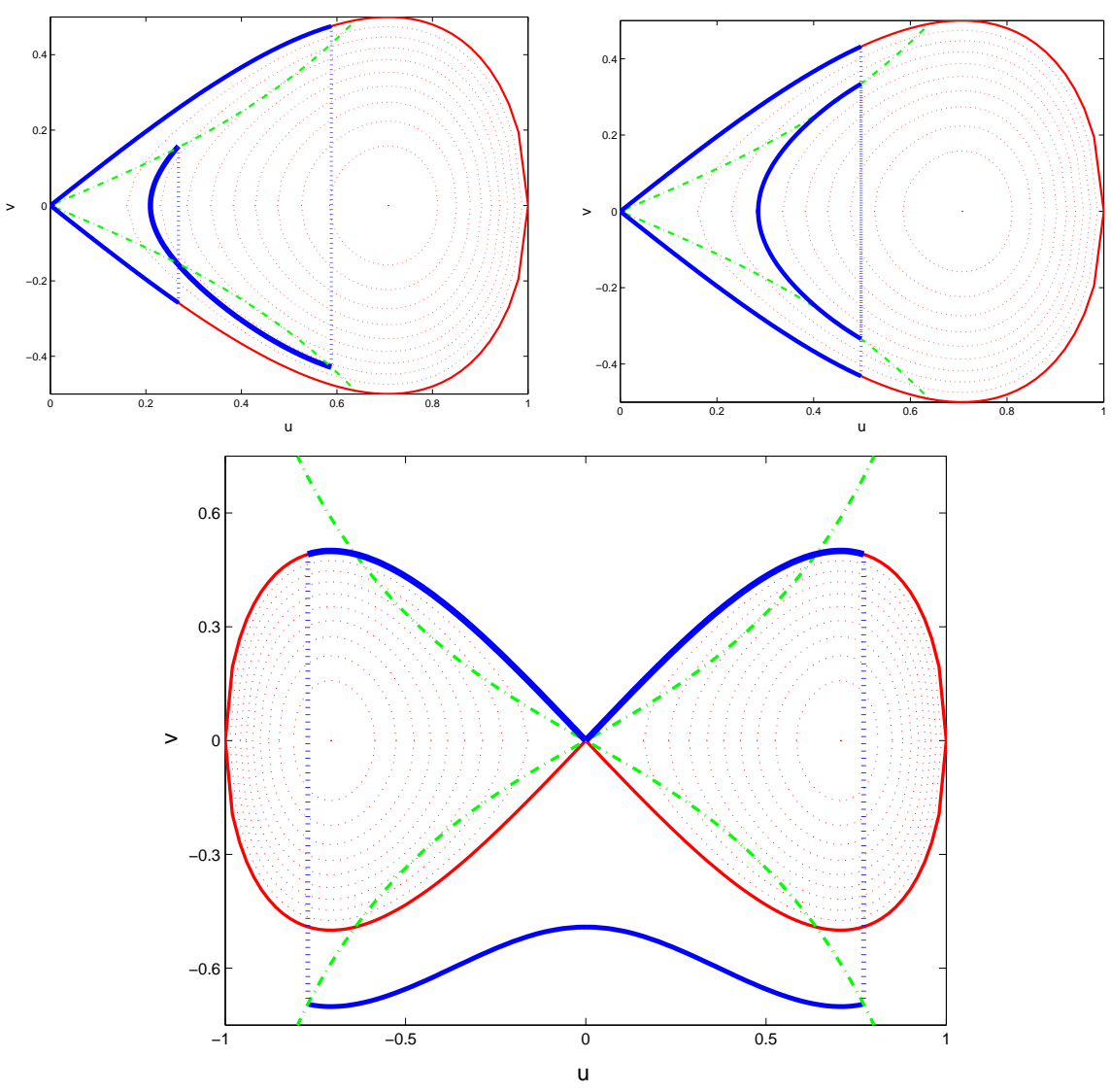

Figure 2: Standing waves of different forms are found for $\epsilon=1, L=2$ and $\lambda=$ 0.65 . Here we see a symmetric wave, an asymmetric wave and an antisymmetric wave. 
For the linear system, a bounded solution again grows from $(0,0)$ along the unstable subspace, jumps to some transient at the first well, evolves further according to the linear system and then must jump exactly to the stable subspace at the second well in order to decay to $(0,0)$ as $z \rightarrow \infty$. For a fixed value of $L$ and $\epsilon$ in this system, we can determine the values of $\lambda$ for which standing waves exist. This is pursued below.

First, the unstable subspace at $(0,0)$ is given as the eigenvector of the matrix

$$
\left(\begin{array}{ll}
0 & 1 \\
1 & 0
\end{array}\right)
$$

associated with the eigenvalue of positive real part. This is the vector $(1,1)$ and so any point in the unstable subspace can be written as $(\phi, \phi)$. Similarly, the stable subspace is spanned by the vector $(1,-1)$. The jump at the first well is then $J:(\phi, \phi) \rightarrow\left(\phi,\left(1-\frac{\epsilon}{\lambda}\right) \phi\right)$. Note that this jump will take us to one of the interior transient curves (inside the homoclinic loop in phase space) when $\lambda>\epsilon / 2$ and to one of the exterior transient curves (outside the homoclinic loop) when $\lambda<\epsilon / 2$.

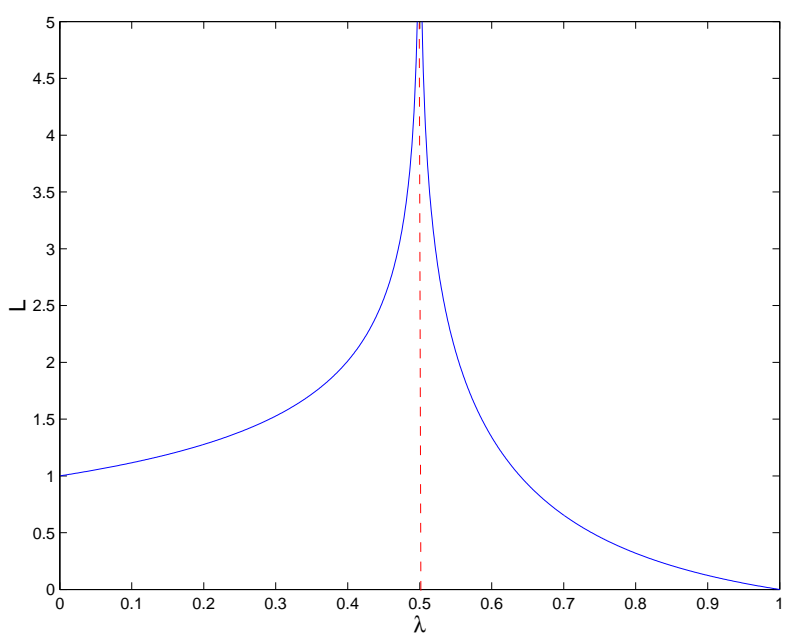

Figure 3: $\lambda$ vs. $L$ for $\epsilon=1$. Symmetric states exist for any $\lambda>\epsilon / 2$, while for $\lambda<\epsilon / 2$, antisymmetric states exist only for well-separation $L>\epsilon^{-1}$.

In this infinitesimal amplitude limit, we can find the standing waves of this system explicitly. Writing the landing point of the first jump in terms of the eigenbasis yields

$$
\left(\phi,\left(1-\frac{\epsilon}{\lambda}\right) \phi\right)=\phi\left(1-\frac{\epsilon}{2 \lambda}\right)(1,1)+\phi \frac{\epsilon}{2 \lambda}(1,-1) .
$$


Because the system is linear in this limit, we ignore the $\phi$-terms from here on. Evolving this point forward for time $2 \lambda L$ brings us to the point

$$
\begin{aligned}
T_{2 \lambda L}\left(\left(1,1-\frac{\epsilon}{\lambda}\right)\right) & =T_{2 \lambda L}\left(\left[1-\frac{\epsilon}{2 \lambda}\right](1,1)+\frac{\epsilon}{2 \lambda}(1,-1)\right) \\
& =\left[1-\frac{\epsilon}{2 \lambda}\right] \mathrm{e}^{2 \lambda L}(1,1)+\frac{\epsilon}{2 \lambda} \mathrm{e}^{-2 \lambda L}(1,-1) \\
& =\left(\mathrm{e}^{2 \lambda L}-\frac{\epsilon}{2 \lambda}\left[\mathrm{e}^{2 \lambda L}-\mathrm{e}^{-2 \lambda L}\right], \mathrm{e}^{2 \lambda L}-\frac{\epsilon}{2 \lambda}\left[\mathrm{e}^{2 \lambda L}+\mathrm{e}^{-2 \lambda L}\right]\right) .
\end{aligned}
$$

Now, the solution we are tracing will represent a standing wave if and only if this point is taken to the stable subspace of zero at the second jump. By the jump conditions at $z= \pm \lambda L$ and the fact that points on the unstable manifold are of the form $(\phi,-\phi)$, such points have the form

$$
J^{-1}\left(W^{s}\right)=\left(\phi,\left(\frac{\epsilon}{\lambda}-1\right) \phi\right) .
$$

Checking to see if the point (3.3) is in this form yields the following equation

$$
(\epsilon-\lambda)\left(\mathrm{e}^{2 \lambda L}-\frac{\epsilon}{2 \lambda}\left(\mathrm{e}^{2 \lambda L}-\mathrm{e}^{-2 \lambda L}\right)\right)-\lambda\left(\mathrm{e}^{2 \lambda L}-\frac{\epsilon}{2 \lambda}\left(\mathrm{e}^{2 \lambda L}+\mathrm{e}^{-2 \lambda L}\right)\right)=0 .
$$

Some algebraic manipulation shows that this equation is equivalent to

$$
L=\frac{1}{2 \lambda} \ln \left(\frac{ \pm \epsilon}{2 \lambda-\epsilon}\right) \text {. }
$$

It may be more natural to consider $\lambda$ as a function of $L$ rather than vice versa; this would allow us to determine for which values of $\lambda$ (frequencies $-\lambda^{2}$ ) stationary waves exist. This can be given implicitly as

$$
\lambda=\frac{\epsilon}{2}\left(1 \pm \epsilon \mathrm{e}^{-2 \lambda L}\right)
$$

In particular, for $\lambda^{2}$ to be real and positive, we need the argument of the log to be positive and therefore we have the following two solutions

$$
\begin{array}{ll}
0<\lambda<\epsilon / 2 & L_{\text {anti }}=\frac{1}{2 \lambda} \ln \left(\frac{\epsilon}{\epsilon-2 \lambda}\right) \\
\epsilon / 2<\lambda<\epsilon & L_{\text {sym }}=\frac{1}{2 \lambda} \ln \left(\frac{\epsilon}{2 \lambda-\epsilon}\right) .
\end{array}
$$

The antisymmetric solutions exist only for $L>\epsilon^{-1}$ because the function $L_{\text {anti }}(\lambda)$ is monotone increasing on $[0, \epsilon / 2)$, with minimum value $\epsilon^{-1}$ attained at $\lambda=0$. The two branches of solutions (symmetric and antisymmetric) are shown in figure 3 The results are summarized in the following proposition:

Proposition 2. In the small amplitude limit, with the wells separated by any positive distance $L>1 / \epsilon$, there exists a symmetric standing wave with $\epsilon / 2<$ 
$\lambda(L)<\epsilon$ and an antisymmetric wave with $0<\lambda(L)<\epsilon / 2$. These states are unique (up to linear scaling) and no other standing waves exist. Finally, for $\epsilon L$ sufficiently large,

$$
\lambda_{ \pm} \sim \frac{\epsilon}{2}\left(1 \pm \mathrm{e}^{-\epsilon L}\right), \quad \lambda_{+}-\lambda_{-} \sim \epsilon \mathrm{e}^{-\epsilon L}
$$

\subsection{Nonlinear regime; solutions with larger amplitude.}

For solutions with nontrivial amplitude, things get much more interesting (as we have seen in our initial numerical search). Because of the periodicity of the transient states, there are many possible solutions for this system (especially for larger values of $L$ ). We show particularly that in addition to the symmetric pulse (and the antisymmetric pulse, for $L$ values at which it exists) described above, there is also an asymmetric pulse that bifurcates away from the symmetric pulse at a particular value of the boson number $\mathcal{N}$. (These results follow for a focusing nonlinearity, $g<0$. In the defocusing case, $g<0$, an asymmetric pulse can be seen to bifurcate from the antisymmetric solution.)

For fixed values of the parameters $L$ and $\epsilon$, we will consider what sorts of positive-valued standing waves exist for varying values of $\lambda$. These solutions can be determined by adapting the previous search technique in the following way:

1. For this system, the branch of the unstable manifold in the first quadrant is given by the explicit expression

$$
W^{u}=\left\{(u, v): v=u \sqrt{1-u^{2}}, 0 \leq u \leq 1\right\} .
$$

We now search for standing waves by evolving this entire curve via the matching conditions and the evolution equation.

2. For a particular value of $\lambda$, application of the matching conditions at the first defect yields the curve

$$
J\left(W^{u}\right)=\left\{(u, v): v=u\left(\sqrt{1-u^{2}}-\frac{\epsilon}{\lambda}\right)\right\} ;
$$

see the dashed curve in figure 2,

3 . We then evolve this curve for time $2 \lambda L$, yielding the curve

$$
T_{2 \lambda L}\left(J\left(W^{u}\right)\right) .
$$

4. Finally, we compare the evolved curve $T_{2 \lambda L}\left(J\left(W^{u}\right)\right)$ with the set of points that jump to the stable manifold at the second defect

$$
J^{-1}\left(W^{s}\right)=\left\{(u, v): v=-u\left(\sqrt{1-u^{2}}-\frac{\epsilon}{\lambda}\right)\right\} .
$$


This agrees with (3.4) in the linear limit. Notice that the set $J^{-1}\left(W^{s}\right)$ is simply the reflection of the set $J\left(W^{u}\right)$ through the $v$-axis. Each nontrivial point of intersection of the two sets $T_{2 \lambda L}\left(J\left(W^{u}\right)\right)$ and $J^{-1}\left(W^{s}\right)$ represents a standing wave of this system.

Remark 3. Because the curve $J\left(W^{u}\right)$ intersects many of the periodic transients more than once, it seems plausible that there may be non-symmetric solutions for some values of $L$. We will make that clear by pursuing the strategy above.

Proposition 4. Let $\epsilon$ and $L$ remain fixed. For all $\lambda>\lambda_{\text {sym }}$ (where $\lambda_{\text {sym }}$ is the value of $\lambda$ for which a symmetric solution exists in the linear limit, as in (3.6)), there is at least one symmetric solution standing wave.

Proof. This is a simple argument regarding the continuity of the curves $J^{-1}\left(W^{s}\right)$ and $T_{2 \lambda L}\left(J\left(W^{u}\right)\right)$. We consider only the portions of these curves interior to the homoclinic orbit of the autonomous system. The portion of $J\left(W^{u}\right)$ inside this loop has one endpoint at $(0,0)$ and the other endpoint on the curve $W^{s}$ in the lower half-plane; see figure 4

For $\lambda>\lambda_{\text {sym }}$, the initial portion of the curve $T_{2 \lambda L}\left(J\left(W^{u}\right)\right)$ leaving $(0,0)$ is between $J^{-1}\left(W^{s}\right)$ and $W^{u}$ - in particular, above the curve $J^{-1}\left(W^{s}\right)$. However, the other end must remain on the invariant manifold $W^{s}$, and this manifold lies below the curve $J^{-1}\left(W^{s}\right)$. Therefore, since $T_{2 \lambda L}\left(J\left(W^{u}\right)\right)$ is a continuous curve, it must intersect the curve $J^{-1}\left(W^{s}\right)$ in at least one point. Therefore there is at least one standing wave in this system for all $\lambda>\lambda_{\text {sym }}$.

Finally, notice that if there is only one point of intersection, then the standing wave corresponding to this intersection point will be a symmetric function of position. This follows from the reversibility of the autonomous portion of the evolution equation.

Knowing that one or more standing waves exist, we now look into the forms that such waves take. In the following, we will look for symmetric standing waves that are concentrated at the defects; in particular, we hope to find profiles that achieve their maximum values at the two defects. We take a slight change of view in the following, considering $\epsilon$ and $\lambda$ as fixed and asking for which values of the spacing parameter $L$ do such standing waves exist?

Proposition 5. Let $\epsilon$ and $\lambda$ be fixed parameters. There is a symmetric solution concentrated at the defects in each of the following regimes:

1. For $\frac{\epsilon}{2}<\lambda<\epsilon$, there is such a solution for all $L>L_{\mathrm{sym}}(\lambda)=\frac{1}{2 \lambda} \ln \left(\frac{\epsilon}{2 \lambda-\epsilon}\right)$.

2. For $\epsilon<\lambda<\sqrt{2} \epsilon$, there is such a solution for all values of $L$.

3. For $\lambda=\sqrt{2} \epsilon$, there is such a solution for all $L>\frac{1}{2 \epsilon}\left(\tan ^{-1} 2\right)$. 

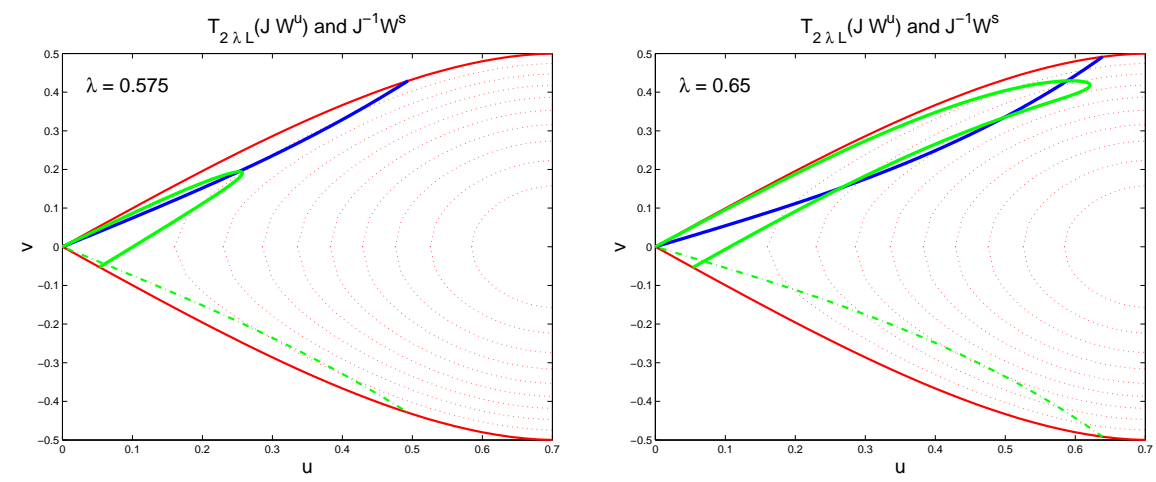

Figure 4: For all values of $\lambda>\lambda_{\text {sym }}$ there is an intersection of $T_{2 \lambda L}\left(J\left(W^{u}\right)\right)$ and $J^{-1}\left(W^{s}\right)$ - and such an intersection represents a standing wave. As $\lambda$ increases, more intersections may arise.

Proof. We will prove this proposition by considering the amount of "time" it takes each point on the curve $J\left(W^{u}\right)$ (from the point where the curve enters the lower half plane until it meets the manifold $W^{s}$ ) to reach its reflection on the curve $J^{-1}\left(W^{s}\right)$. For fixed value $2 L$, we show there is a point on $J\left(W^{u}\right)$ which is mapped to its reflection on $J^{-1}\left(W^{s}\right)$ in time $2 L$. We can define the function $L(u)$ as the "time" it takes the solution beginning at a point $\left(u, u\left(\sqrt{1-u^{2}}-\epsilon / \lambda\right)\right)$ (meeting the previous parenthetical requirement) to reach the symmetric point $\left(u,-u\left(\sqrt{1-u^{2}}-\epsilon / \lambda\right)\right)$.

1. The result follows quickly in the regime $\frac{\epsilon}{2}<\lambda<\epsilon$, by noting that as $u \rightarrow 0^{+}, L(u) \rightarrow L_{s}$ as given in the earlier proposition. Also, as $u \rightarrow$ $\left(\sqrt{1-(\epsilon / 2 \lambda)^{2}}\right)^{-}, L(u) \rightarrow \infty$. A continuity argument completes this case.

2. For $\lambda>\epsilon$ the curve $J\left(W^{u}\right)$ leaves the origin into the upper half plane. It reaches the $u$-axis when $u=\sqrt{1-(\epsilon / \lambda)^{2}}$ and reaches the stable manifold $W^{s}$ when $u=\sqrt{1-(\epsilon / 2 \lambda)^{2}}$. In this regime, it is clear that $L(u) \rightarrow 0$ as $u \rightarrow\left(\sqrt{1-(\epsilon / \lambda)^{2}}\right)^{+}$and $L(u) \rightarrow \infty$ as $u \rightarrow\left(\sqrt{1-(\epsilon / 2 \lambda)^{2}}\right)$

3. When $\lambda=\sqrt{2} \epsilon$, the curve $J\left(W^{u}\right)$ crosses the $u$-axis at the elliptic fixed point $(1 / \sqrt{2}, 0)$. A careful study of the linearization near this fixed point reveals the lower limit given above.

Now we note an important consideration concerning the form of these symmetric pulses. In particular, for fixed values of the parameters $\epsilon$ and $\lambda$, there is a distinguished value of the parameter $L$ so that the curve $J\left(W^{u}\right)$ is tangent 
to the symmetric standing wave as we approach the first defect from the right. The value of the standing wave at each defect can be quickly computed for this threshold value,

$$
u_{\text {thresh }}=\sqrt{\frac{12-\left(\frac{\epsilon}{\lambda}\right)^{2}-\frac{\epsilon}{\lambda} \sqrt{12+\left(\frac{\epsilon}{\lambda}\right)^{2}}}{18}}
$$

and the threshold value of $L, L_{\text {thresh }}$, can then be computed simply by noting the "time" it takes to flow from $\left(u_{\text {thresh }}, v_{\text {thresh }}\right)$ to its reflection $\left(u_{\text {thresh }},-v_{\text {thresh }}\right)$.

In figure 5] we show the energy of the symmetric standing wave at the threshold values of $L$. We will now show that beyond this threshold, there is a pair of asymmetric standing waves (in addition to the symmetric and antisymmetric waves discussed previously).

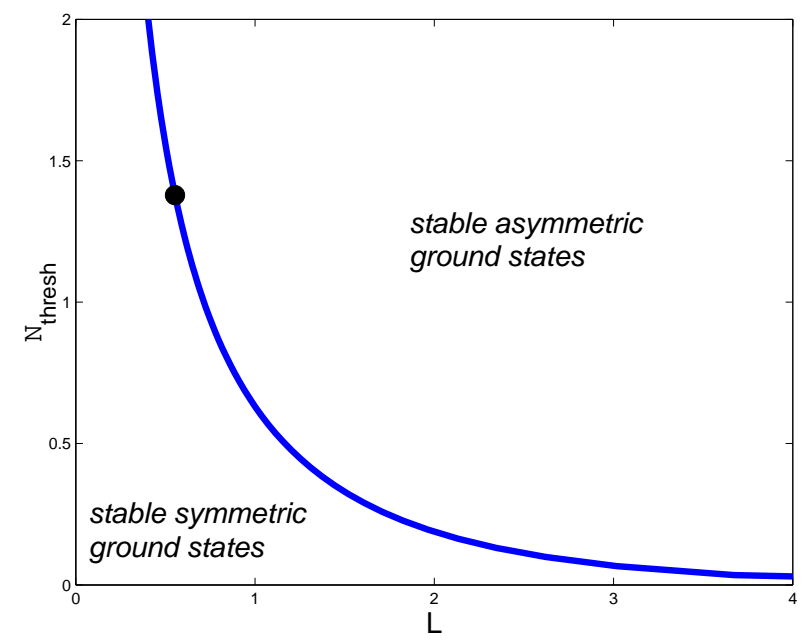

Figure 5: Threshold values of the particle number $\mathcal{N}_{\text {thresh }}$ for varying values of $L$ are shown. At these threshold values, there is a bifurcation of asymmetric pulses (see Proposition 6) and beyond these values, the symmetric standing wave will be unstable (see Theorem 8). The point along the curve marked with a solid circle corresponds to the special value $\lambda=\sqrt{2} \epsilon$, where the point $u_{\text {thresh }}$ is the fixed point in the phase plane. Along the curve to the right of this point, the symmetric standing waves at threshold have the desired two peak profile; to the left, the symmetric standing waves at threshold have a single peak centered between the two wells.

Proposition 6. Let $\epsilon$ and $\lambda$ remain fixed. For $L>L_{\text {thresh }}(\lambda)$, there exists a pair of non-symmetric standing waves. These waves leave the symmetric wave in a pitchfork bifurcation at $L=L_{\text {thresh }}$. 

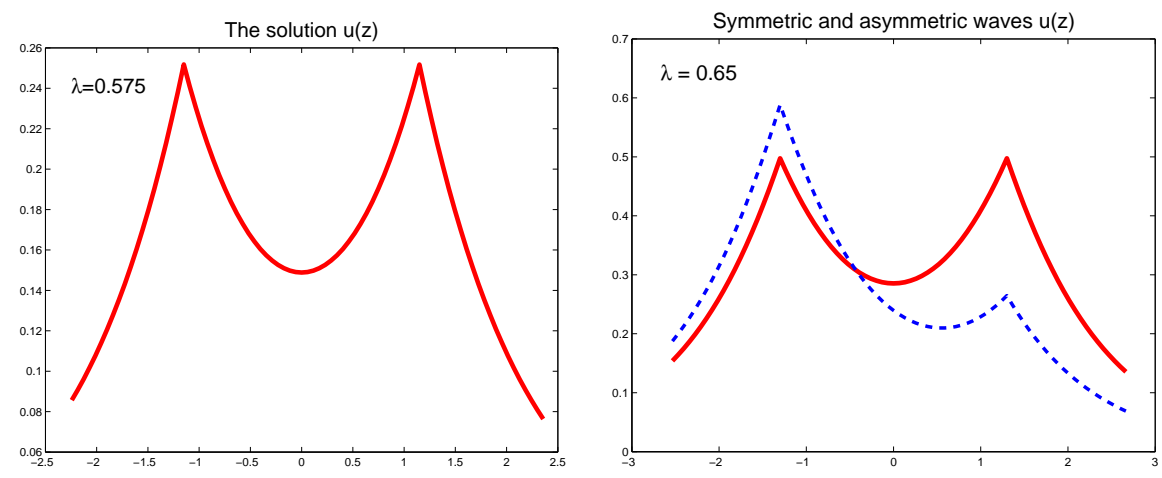

Figure 6: The solutions corresponding to the various intersection points from the phase plane diagram in figure 4 are shown here.

Proof. Consider $\epsilon$ and $\lambda$ fixed and positive, with $L$ so that $L>L_{\text {thresh }}(\lambda)$. For this value of $L$, the symmetric standing wave has a value $\left(u_{0}, v_{0}\right)$, with $u_{0}>u_{\text {thresh }}$, approaching the first defect from the right. This wave then must pass through $J^{-1}\left(W^{s}\right)$ in the phase plane an additional time before the reaching the terminal point at the second defect.

In the phase space, the part of the standing wave between the two defects can be written as the curve

$$
\Gamma_{0}=\left\{T_{z}\left(\left(u_{0}, v_{0}\right)\right): 0 \leq z \leq 2 \lambda L\right\} .
$$

Note that the curve $\Gamma_{0}$ simply traces out the portion of the periodic orbit connecting the symmetric points $\left(u_{0}, v_{0}\right)$ and $\left(u_{0},-v_{0}\right)$.

We consider an arbitrary parameterization $\left(u_{\tau}, v_{\tau}\right), \tau \in[0,1]$ of the portion of the curve $J\left(W^{u}\right)$ between $\left(u_{0}, v_{0}\right)$ and its nontrivial intersection with the stable manifold $W^{s}$, so that $\left(u_{0}, v_{0}\right)$ is as above and $\left(u_{1}, v_{1}\right) \in W^{s}$. Then we can define

$$
\Gamma_{\tau}=\left\{T_{z}\left(\left(u_{\tau}, v_{\tau}\right)\right): 0 \leq z \leq 2 \lambda L\right\},
$$

for all $\tau \in[0,1]$. Notice that the curve $J^{-1}\left(W^{s}\right)$ is transverse to all of the periodic orbits in the phase plane away from the fixed point $(1 / \sqrt{2}, 0)$. Therefore if a curve $\Gamma_{\tau}$ crosses $J^{-1}\left(W^{s}\right)$, it must do so transversely.

Recall that the curve $\Gamma_{0}$ crosses $J^{-1}\left(W^{s}\right)$ prior to its terminal point at the second defect. (This earlier crossing does not represent a standing wave, of course, since it occurs for a value of $z$ not corresponding to a defect.) On the other hand, for $\tau$ sufficiently close to 1 , the curve $\Gamma_{\tau}$ no longer crosses $J^{-1}\left(W^{s}\right)$. Continuity considerations therefore dictate that there must be a value of $\tau$ between 0 and 1 so that $T_{2 \lambda L}\left(\left(u_{\tau}, v_{\tau}\right)\right)$ lies on the curve $J^{-1}\left(W^{s}\right)$. This value clearly does not represent a symmetric state since $u_{0}>u_{\text {thresh }}$, while the first point of intersection is smaller than $u_{\text {thresh }}$.

There is a second non-symmetric standing wave that, in the phase plane, has the form of the wave just found reflected across the $u$-axis. 
This pair of non-symmetric solutions is originally produced in a pitchfork bifurcation when $L=L_{\text {thresh }}$ - at this parameter value the transversality of $J^{-1}\left(W^{s}\right)$ and the periodic orbit carrying the symmetric standing wave is lost.

\section{The eigenvalue problem and stability.}

In the previous section, we found various standing wave solutions of the equation

$$
\mathrm{i} \psi_{t}=-\psi_{x x}-2|\psi|^{2} \psi+\epsilon v(x) \psi
$$

where $v(x)$ is the double well potential $-\delta(x-L)-\delta(x+L)$. In this section, we will analyze the stability of the various branches of solutions constructed, by studying the eigenvalue equation, associated with linearization about a fixed nonlinear bound state.

First we change coordinates into the frame of the standing wave

$$
\psi(x, t)=\mathrm{e}^{\mathrm{i} \lambda^{2} t} \phi(x, t)
$$

and arrive at the equivalent equation

$$
\mathrm{i} \phi_{t}=-\phi_{x x}+\left[\lambda^{2}-\left(2|\phi|^{2}+\epsilon v(x)\right)\right] \phi .
$$

We write this equation in real and imaginary parts $(\phi=v+\mathrm{i} w)$

$$
\begin{aligned}
& v_{t}=-w_{x x}-\left[2\left(v^{2}+w^{2}\right)+\epsilon v(x)\right] w+\lambda^{2} w \\
& w_{t}=v_{x x}+\left[2\left(v^{2}+w^{2}\right)+\epsilon v(x)\right] v-\lambda^{2} v
\end{aligned}
$$

and formally consider small perturbations of the standing wave. Since the standing wave (let's call it $u(x))$ is real-valued, we then write $(v(x, t), w(x, t))=$ $(u(x), 0)+\delta(p(x, t), q(x, t))$ with $\delta$ small. Leading order in $\delta$, yields the linearized dynamics

$$
\begin{aligned}
& p_{t}=-q_{x x}-\left[2 u^{2}+\epsilon v(x)\right] q+\lambda^{2} q \\
& q_{t}=p_{x x}+\left[2 u^{2}+\epsilon v(x)\right] p-4 u^{2} p-\lambda^{2} p \text {. }
\end{aligned}
$$

Setting

$$
\begin{aligned}
& L_{-}=\frac{\mathrm{d}^{2}}{\mathrm{~d} x^{2}}+\left[2 u^{2}+\epsilon v(x)\right]-\lambda^{2}, \\
& L_{+}=\frac{\mathrm{d}^{2}}{\mathrm{~d} x^{2}}+\left[2 u^{2}+\epsilon v(x)\right]+4 u^{2}-\lambda^{2},
\end{aligned}
$$

equation (4.2) can be rewritten as the system

$$
\left(\begin{array}{c}
p \\
q
\end{array}\right)_{t}=\left(\begin{array}{cc}
0 & -L_{-} \\
L_{+} & 0
\end{array}\right)\left(\begin{array}{l}
p \\
q
\end{array}\right)=N\left(\begin{array}{l}
p \\
q
\end{array}\right) .
$$

Of interest is the spectrum of this operator $N$. For the ground state (see section 21), the spectrum of $N$ lies entirely on the imaginary axis and modulo zero modes, 
associated with symmetries, $\exp (t N)$ is bounded on $H^{1}$ and indeed, the solution is nonlinearly stable [17, 13.

A state is unstable if one can exhibit an eigenvalue of the operator $N$ with positive real part. Nonlinear instability is a consequence; see the appendix of [8. Unfortunately, even though the spectrum of $L_{+}$and $L_{-}$can, in principle, be determined using Sturm-Liouville theory, it is not obvious how to construct the spectrum of $N$ using this information. However, this is exactly the operator studied by Jones in [9, in an application to optical pulses in nonlinear waveguides. In that work, Jones interprets the search for a real positive eigenvalue as a shooting problem in the space of Lagrangian planes. Using a winding argument in this space, he derives a relationship between the number of positive eigenvalues of the simpler operators $L_{+}$and $L_{-}$and the number of positive eigenvalues of $N$. Let

$$
\begin{aligned}
& P=\text { number of positive eigenvalues of } L_{+} \text {and } \\
& Q=\text { number of positive eigenvalues of } L_{-} .
\end{aligned}
$$

Then we have 9,8 :

Theorem 7. If $P-Q \neq 0,1$, there is a real positive eigenvalue of the operator $N$.

An alternative, PDE approach to this result was developed by Grillakis 8 .

From Sturm-Liouville theory, $P$ and $Q$ can be determined by considering the behavior of solutions of $L_{+} p=0$ and $L_{-} q=0$, respectively. In particular, $P$ and $Q$ equal the number of nodes of the associated solutions $p$ and $q$. Since $L_{-} q=0$ is satisfied by the standing wave $u(x)$ itself, it quickly follows that

$$
Q=\text { number of zeros of the standing wave } u(x) \text {. }
$$

For all positive-valued pulses, then, $Q=0$.

So we have that if $P>1$, then $N$ has a positive eigenvalue and the standing wave $u(x)$ is unstable. The operator $L_{+}$acts as the equation of variations for (4.1), and a natural interpretation of the equation of variations is that it carries tangent vectors under the flow. So if we initialize $q(x)$ as a vector tangent to the unstable manifold at the point $u(x)$, then we can calculate $P$ by counting the nodes of the resulting solution $q(x)$.

In the context of vectors, a node of $q(x)$ is a vector in the phase plane pointing straight up or down. And so, $P$ can be computed by counting the number of times that the vector initialized tangent to the unstable manifold passes through verticality. In particular, considering only the angle of this vector and not its magnitude, $P$ corresponds to the largest number of integer multiples of $\pi$ through which this vector rotates as it is evolved under the linearized flow.

The only difficulty in the computation is this: because of the jumps at the defects, i.e., the inhomogeneity of the evolution equation, a vector $q(x)$ 
initialized tangent to the nonlinear bound state solution will not remain tangent to this solution once we reach the first defect.

However, using the simple matching conditions for these vectors at the wells, we have the following theorem:

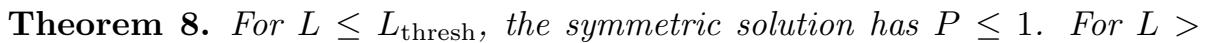

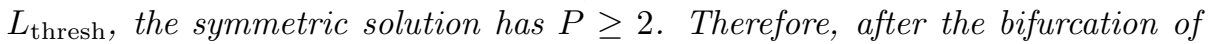
the asymmetric waves, the symmetric wave is unstable.

Proof. The result follows simply by evolving a vector (initialized as a tangent vector to the upper branch of the homoclinic) around each standing wave under the influence of the equation of variations. This can be visualized by noting that, at the first defect, the tangent vector to the unstable manifold $W^{u}$ is taken to a vector that is tangent to the curve $J\left(W^{u}\right)$. The observation of instability is then reduced to simply noting the manner in which this landing curve $J\left(W^{u}\right)$ intersects the periodic curve in the phase plane representing the standing wave between the defects.

1. At the threshold value $L=L_{\text {thresh }}$ itself, the curve $J\left(W^{u}\right)$ is tangent to the transient to which the solution jumps at the first well. The variational solution, then, will still be tangent to the transient curve (although pointing backwards). Hence, we can continue to trace the solution exactly by following the tangent vector around this transient to the second jump. Then, as symmetry dictates, this vector is taken to a tangent vector to $W^{s}$ at the second jump. This vector is seen to pass through verticality only once (at $t=0$ ) and hence has $P=1$.

2. For $L<L_{\text {thresh }}$, the vector jumps through a smaller angle at each well than it does at the bifurcation point itself. At the first defect, it then points along the curve $J\left(W^{u}\right)$, which in this case points up and into the internal ellipse. Because solutions are unique, the tangent vector to this ellipse provides a bound for our solution as it evolves, and the vector in question must retain its orientation with respect to the tangent to the ellipse; in particular, it rotates clockwise and must be pointing "below" the ellipse at the second defect. However, the vector that is taken to the curve $W^{s}$ is the vector tangent to $J^{-1}\left(W^{s}\right)$, and comparing these two vectors we quickly see that $P \leq 1$ in this case.

3. For $L<L_{\text {thresh }}$, the vector jumps through a larger angle at the first defect and now points along $J\left(W^{u}\right)$ down and out of the internal ellipse, and so the tangents to the ellipse now provide a bound on the other side. At the second well, the solution must still point out of the ellipse and comparison with the vector tangent to $J^{-1}\left(W^{s}\right)$ forces $P \geq 2$ in this case.

These three cases are sketched in figure 7 

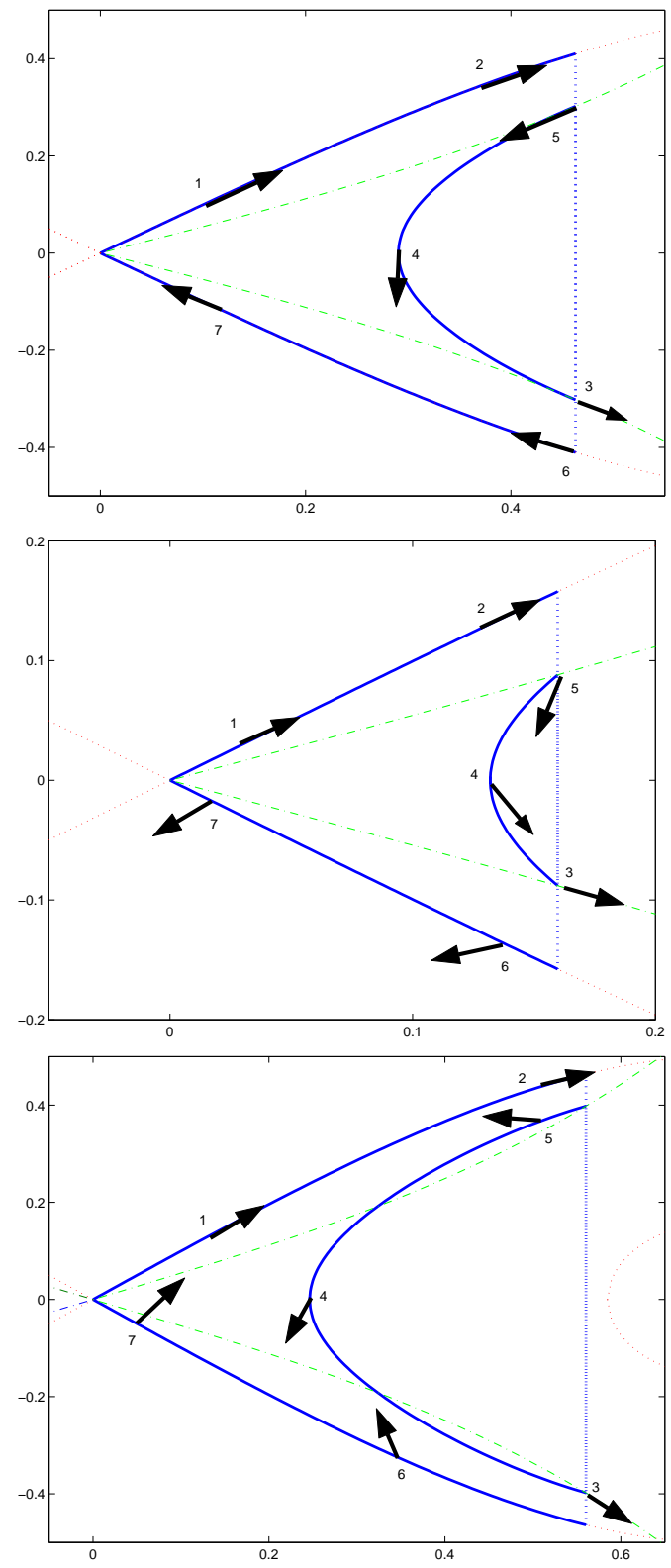

Figure 7: The evolution of the tangent vector is shown for solutions with varying values of $L$. In the first figure $L=L_{\text {thresh }}$ and the evolved vector is parallel to the solution throughout its evolution. In the second figure $L<L_{\text {thresh }}$ and the vector moves through a smaller angle at each defect and we see $P \leq 1$. When $L>L_{\text {thresh }}$ as in the third figure, the vector moves through a larger angle at each defect and forces $P \geq 2$. 


\section{A Wellposedness of Gross-Pitaevskii / NLS}

Structural properties of NLS: The Gross-Pitaevskii / NLS equation is a Hamiltonian system, which can be written in the form:

$$
\mathrm{i} u_{t}=\frac{\delta}{\delta \bar{u}} \mathcal{H}_{\mathrm{GP}}[u, \bar{u}],
$$

where $\mathcal{H}_{\mathrm{GP}}[u, \bar{u}]$ denotes the Hamiltonian (1.5). Invariance with respect to timetranslations implies that $\mathcal{H}_{\mathrm{GP}}[u, \bar{u}]$ is conserved by the flow generated by (A.1). Additionally, invariance under the transformation $u \mapsto e^{i \xi} u, \xi \in R^{1}$ implies that $\mathcal{N}[u, \bar{u}]$, the $L^{2}$ norm defined in (1.6), is conserved.

For the spatially translation invariant case, NLS has the Galilean invariance:

$$
u(x, t) \mapsto u(x-s t, t) \mathrm{e}^{\mathrm{i}\left(x v-\frac{1}{2} s^{2} t\right)}, s \in R^{1} .
$$

Well-posedness theory: We present a sketch of the existence and uniqueness of solutions to (1.3); see also [7]. The functionals $\mathcal{H}_{\mathrm{GP}}[\cdot]$ and $\mathcal{N}[\cdot]$ are well defined on $H^{1}\left(R^{1}\right)$, the space of functions $f$, for which $f$ and $\partial_{x} f$ are square integrable. It is therefore natural to construct the flow for initial data of class $H^{1}$. In fact, it can be shown that, for initial conditions $u_{0}=u(x, t=0) \in H^{1}\left(R^{1}\right)$, there exists a unique global solution of NLS, $u \in C^{0}\left(R^{1} ; H^{1}(R)\right)$, in the sense of the equivalent integral equation:

$$
\begin{gathered}
u(t)=U(t) u_{0}+2 \mathrm{i} \int_{0}^{t} U(t-s)|u(s)|^{2} u(s) \mathrm{d} s, \\
U(t) \equiv \exp (-\mathrm{i} H t), H \equiv-\partial_{x}^{2}+v(x) .
\end{gathered}
$$

The spectral decomposition of $H$ is known explicitly 2 and can be used to construct $U(t)$ explicitly.

To show the existence of a solution to A.3a in $H^{1}$, we must show the existence of a $C^{0}\left(R^{1} ; H^{1}(R)\right)$ fixed point of the mapping $u(x, t) \mapsto J[u](x, t)$, given by the right hand side of A.3a).

We now outline the key ingredients of the proof. To bound $J[u]$ and its first derivative in $L^{2}$, we introduce the operator $\mathcal{A}=I+P_{c} H$, where $P_{c}$ denotes the projection onto the continuous spectral part of $H$. Note that $\mathcal{A}$ is a nonnegative operator, since the continuous spectrum of $H$ is the nonnegative real half-line. Moreover, we expect $\left\|\mathcal{A}^{\frac{1}{2}} f\right\| \sim\|f\|_{H^{1}} \equiv\left\|\left(I-\partial_{x}^{2}\right)^{\frac{1}{2}} f\right\|_{L^{2}}$. In fact, we shall use that the following operators are bounded from $L^{2}$ to $L^{2}$ :

$$
\mathcal{A}^{\frac{1}{2}}\left(I-\partial_{x}^{2}\right)^{-\frac{1}{2}}, \mathcal{A}^{-\frac{1}{2}}\left(I-\partial_{x}^{2}\right)^{\frac{1}{2}} .
$$

This follows from the boundedness of the wave operators on $H^{1}$ [16]. Therefore, we have an equivalence of norms

$$
C_{1}\|f\|_{H^{1}} \leq\left\|\mathcal{A}^{\frac{1}{2}} f\right\|_{L^{2}} \leq C_{2}\|f\|_{H^{1}} .
$$

Our formulation a.3a and introduction of $\mathcal{A}$ are related to the nice property that $\mathcal{A}$, and hence also functions of $\mathcal{A}$, commute with the propagator $\exp (-\mathrm{i} H t)$. 
We shall also use the Sobolev inequality:

$$
|f(x)|^{2} \leq C\|f\|_{L^{2}}\left\|\partial_{x} f\right\|_{L^{2}}
$$

and the Leibniz rule [10]:

$$
\left\|\left(I-\partial_{x}^{2}\right)^{\frac{1}{2}}(f g)\right\| \leq C\left(\|f\|_{L^{\infty}}\left\|\left(I-\partial_{x}^{2}\right)^{\frac{1}{2}} g\right\|_{L^{2}}+\left\|\left(I-\partial_{x}^{2}\right)^{\frac{1}{2}} f\right\|_{L^{2}}\|g\|_{L^{\infty}}\right) .
$$

Since $U(t)$ is unitary in $L^{2}$, we have

$$
\begin{aligned}
& \left\|\mathcal{A}^{\frac{1}{2}} J[u]\right\|_{L^{2}} \\
& \leq\left\|\mathcal{A}^{\frac{1}{2}} u_{0}\right\|_{L^{2}}+\gamma \int_{0}^{t}\left\|\mathcal{A}^{\frac{1}{2}}|u(s)|^{2} u(s)\right\|_{L^{2}} d s \\
& =\left\|\mathcal{A}^{\frac{1}{2}} u_{0}\right\|_{L^{2}}+\gamma \int_{0}^{t}\left\|\left(\mathcal{A}^{\frac{1}{2}}\left(I-\partial_{x}^{2}\right)^{-\frac{1}{2}}\right) \cdot\left(\left(I-\partial_{x}^{2}\right)^{\frac{1}{2}}|u(s)|^{2} u(s)\right)\right\|_{L^{2}} d s .
\end{aligned}
$$

By (A.4, A.5), and (A.6),

$$
\|J[u](t)\|_{H^{1}} \leq C\|\mathcal{A} J[u]\|_{L^{2}} \leq C_{1}\left\|u_{0}\right\|_{H^{1}}+C_{2} T \sup _{s \in[0, T]}\|u(s)\|_{H^{1}}^{3} .
$$

Now assume that $u$ is such that $\sup _{s \in[0, T]}\|u(s)\|_{H^{1}} \leq 2 C_{1}$. Then, by (A.8), choosing $T<T_{1}$ sufficiently small, $\sup _{s \in[0, T]}\|J[u](s)\|_{H^{1}} \leq 2 C_{1}\left\|u_{0}\right\|_{H^{1}}$ and so

$$
\|J[u](t)\|_{H^{1}} \leq C_{1}\left\|u_{0}\right\|_{H^{1}}+C_{2} T\left(2 C_{1}\left\|u_{0}\right\|_{H^{1}}\right)^{3} .
$$

It follows that for $0<T<T_{1}$ sufficiently small, the transformation $J[\cdot]$ maps a ball $C^{0}\left([0, T] ; H^{1}(R)\right)$ into itself. A similar calculation shows that

$$
\|J[u](t)-J[v](t)\|_{H^{1}} \leq K T\left(2 C_{1}\left\|u_{0}\right\|_{H^{1}}\right)^{2} \sup _{s \in[0, T]}\|u(s)-v(s)\|_{H^{1}},
$$

and therefore for $0<T<T_{2} \leq T_{1}$, the transformation $J[\cdot]$ is a contraction on this ball. Therefore, $J[\cdot]$ has a unique fixed point in $C^{0}\left([0, T] ; H^{1}(R)\right)$ for $T$ sufficiently small and local existence in time of the flow follows. Global existence in time follows from the a priori bound on the $H^{1}$ norm of the solution implied by the time-invariance of $L^{2}$ norm, $\mathcal{N}$, and the Hamiltonian, $\mathcal{H}_{\mathrm{GP}}$.

\section{Acknowledgements}

This work was initiated while R.K. Jackson participated in the Bell Labs / Lucent Student Intern Program. R.K. Jackson would like to thank C.K.R.T. Jones for fruitful discussions and guidance, and also gratefully acknowledges the current support of the National Science Foundation under award DMS-0202542.

\section{References}

[1] R. Adami, C. Bardos, F. Golse and A. Teta, Towards a rigorous derivation of the cubic NLSe in dimension one, preprint, 2003. 
[2] S. Albeverio, F. Gesztesy, R. Hëgh-Krohn and H. Holden, Solvable models in quantum mechanics (Springer Verlag, New York, 1988).

[3] W. H. Aschenbacher, J. Fröhlich, G. M. Graf, K. Schnee and M. Troyer, Symmetry breaking regime in the nonlinear Hartree equation, J. Math. Phys. 43 (2002) 3879-3891.

[4] F. Dalfovo, S. Giorgini, L. P. Pitaevskii and S. Stringari, Theory of BoseEinstein condensation in trapped gases, Rev. Mo. Phys. 71 (1999) 463-512.

[5] L. Erdös, H. T. Yau, Derivation of the nonlinear Schrödinger equation from a many body Coulomb system, Adv. Theor. Math. Phys. 5 (2001) 11691205.

[6] Yu. B. Gaididei, S. F. Mingaleev and P. L. Christiansen, Curvature-induced symmetry breaking in nonlinear Schrödinger models, Phys. Rev. E 62 (2000) R53-55.

[7] R.H. Goodman, P.J. Holmes and M.I. Weinstein, Strong NLS soliton-defect interactions, submitted to Physica D, 2003

[8] M. Grillakis, Analysis of the linearization around a critical point of an infinite dimesional Hamiltonian system, Commun. Pure Appl. Math. 43 (1990) 299-333.

[9] C. K. R. T. Jones, Instability of standing waves for non-linear Schrödingertype equations, Ergodic Theory Dynam. Systems 8 (1988) 119-138.

[10] T. Kato and G. Ponce, Commutator estimates and the Euler and NavierStokes equations, Comm. Pure Appl. Math. 41 (1988) 891-907.

[11] E. H. Lieb, R. Seiringer and J. Yngvason, A rigorous derivation of the Gross-Pitaevskii energy functional for a two-dimensional Bose gas. Commun. Math. Phys. 224 (2001) 17-31.

[12] K. W. Mahmud, J. N. Kutz and W. P. Reinhardt, Bose-Einstein condensates in a one-dimensional double square well: Analytical solutions of the nonlinear Schrödinger equation, Phys. Rev. A 66 (2002) 063607.

[13] H. A. Rose and M. I. Weinstein, On the bound states of the nonlinear Schrödinger equation with a linear potential, Physica D 30 (1988) 207218.

[14] A. Soffer and M. I. Weinstein, Resonances and radiation damping in Hamiltonian nonlinear wave equations, Invent. Math. 136 (1999) 9-74.

[15] A. Soffer and M. I. Weinstein, Selection of the ground state in nonlinear Schrödinger equations, submitted, 2001.

[16] R. Weder, The $W_{k, p}$ continuity of the Schrödinger wave operators on the line, Commun. Math. Phys. 208 (1999) 507-520. 
[17] M. I. Weinstein, Modulational stability of ground states of nonlinear Schrödinger equations, SIAM J. Math. Anal. 16 (1985) 472-491.

[18] M. I. Weinstein, Lyapunov stability of ground states of nonlinear dispersive evolution equations, Comm. Pure Appl. Math. 39 (1986) 51-68. 